\title{
Isolating approaches: How middle-division physics students coordinate forms and representations in complex algebra
}

\author{
Emily M. Smith, ${ }^{1,2, *}$ Justyna P. Zwolak, ${ }^{3}$ and Corinne A. Manogue ${ }^{1}$ \\ ${ }^{1}$ Department of Physics, Oregon State University, Corvallis, Oregon 97331, USA \\ ${ }^{2}$ Laboratory of Atomic and Solid State Physics, Cornell University, Ithaca, New York 14853, USA \\ ${ }^{3}$ Joint Center for Quantum Information and Computer Science, University of Maryland, \\ College Park, Maryland 20742, USA
}

(Received 19 December 2017; revised manuscript received 8 April 2019; published 10 June 2019)

\begin{abstract}
Mathematical reasoning with algebraic and geometric representations is essential for success in upperdivision and graduate-level physics courses. Complex algebra requires student to fluently move between algebraic and geometric representations. By designing a task for middle-division physics students to translate a geometric representation to several algebraic representations, we identified students' facilities and difficulties with complex number algebra. Students were successful when they isolated approaches, which was characterized by students' use of circle trigonometry, triangle trigonometry, the Pythagorean theorem, and the square of the norm approaches. When students were not successful, they inappropriately mixed approaches to the problem, including failing to distinguish between triangle and circle trigonometry.
\end{abstract}

DOI: 10.1103/PhysRevPhysEducRes.15.010138

\section{INTRODUCTION}

Expert physicists regularly use geometric and algebraic reasoning to understand the physical world. The ability to simultaneously and interchangeably use and reason with algebraic and geometric representations is crucial for success in all physics subdisciplines. In physics courses, students use mathematics to model physical phenomena, which may be strikingly different from the abstract nature of, or procedural emphasis in, many prerequisite mathematics courses. To become knowledgeable physicists, students must supplement the procedural knowledge developed in their mathematics courses with conceptual knowledge of the physical world.

Complex algebra is a prime example of straightforward mathematics that demands sophisticated algebraic and geometric reasoning, especially within physics contexts. In this paper, we refer to algebraic, or analytic, reasoning when students manipulate symbols to find meaning [1]. Geometric reasoning is exhibited by students who reason using geometric representations such as shapes, graphs, and pictures [1]. Previous research demonstrated that experts readily engaged in both algebraic and geometric reasoning in complex algebra problems by easily selecting and changing between forms and representations of complex

\footnotetext{
*emsmith@cornell.edu
}

Published by the American Physical Society under the terms of the Creative Commons Attribution 4.0 International license. Further distribution of this work must maintain attribution to the author(s) and the published article's title, journal citation, and DOI. numbers and functions [2,3]. However, a number of previous studies have investigated students' and instructors' understanding of complex algebra in mathematics contexts [4-11], and these studies suggest the development of reasoning with both algebraic and geometric representations of complex numbers and functions is gradual.

Studies repeatedly demonstrated that arithmetic with complex numbers was not trivial for lower-division mathematics students [4-8], including those who planned to teach or have taught high school mathematics $[4,6,9]$. Students often exhibited difficulties with simple complex number arithmetic $[4,6]$, but their difficulties may have lessened with the introduction of geometric reasoning $[4,6]$, possibly mediated by physical enactment [6]. Furthermore, students' abilities to perform algebraic arithmetic may have assisted in their development of geometric reasoning [6]. Some studies suggest that difficulties possibly originated from students' struggles to trust complex number arithmetic [4] or emotions evoked by the labels associated with complex algebra (e.g., imaginary and complex) [7].

Even upper-division mathematics students may not spontaneously engage in interchangeable algebraic and geometric reasoning with complex arithmetic. When solving a series of arithmetic problems, students enrolled in complex analysis persisted in their use of algebraic reasoning rather than drawing on geometric reasoning [5]. Similarly, when prompted to provide geometric reasoning, upper-division students first reasoned algebraically before shifting to geometric reasoning [11].

Physics students do not typically require the extensive knowledge of complex analysis demanded of upperand graduate-level mathematics students. However, to be 
successful in most courses, physics students need to be proficient in several aspects of complex algebraperforming calculations and changing between and selecting appropriate forms-using numbers, symbols, and functions. Persistent difficulties with complex algebra may cause extensive issues in learning physics in courses where complex algebra is pervasive and necessary, such as quantum mechanics. In order for students to appropriately model physical situations, even as simple as damped and driven oscillations, they must coordinate algebraic and geometric representations of complex functions in parallel with a physical situation. However, review of mathematics curricula at Oregon State University (OSU) reveals that, although complex algebra appears in many upper-division mathematics courses, explicit instruction is often missing from necessary prerequisite courses for middle-division physics courses (e.g., differential equations and linear algebra).

Previous research hints that upper-division and graduate physics students consistently encounter difficulties with complex algebra tasks embedded within physics contexts [12-16]. Upper- and graduate-level physics students exhibit some complex algebra difficulties that are similar to lower-division mathematics students but often with the added sophistication of dealing with complex functions and physical context. Gupta, Redish, and Hammer described graduate students' treatment of a complex function in a plasma physics context; students were unsuccessful when they expressed a function in a form visually similar to the rectangular form of a complex number, $x+i y$, despite an embedded complex variable in the function [12]. Sadaghiani found many upper-division students may not distinguish between functions of the form $e^{k x}$ and $e^{i k x}$ [14]. Loverude and Li found that physics students have difficulty graphing functions of the form $f(t)=A e^{i \omega t}$ when plotting the real part [13]. When students assessed relative phases in quantum mechanics, Close et al. found successful students used embodied geometric reasoning rather than relying on algebraic approaches [15]. Wan et al. identified that middle-division students encounter difficulties relating complex-valued functions to the geometry of the complex plane [16].

To our knowledge, this study is the first that specifically focuses on physics students' abilities with complex number algebra without physical context or complex functions. Previous studies of physics students incorporated physical context or mathematics beyond simple complex number algebra, which introduces complications with disentangling complex algebra difficulties from other physics or mathematics difficulties. This study is informed by our previous work analyzing students' calculational abilities on quizzes and exams, which showed some middle-division physics students at OSU encountered difficulties with simple complex algebra tasks and that some difficulties persisted into upper-division courses [17]. Based on these findings from mathematics and physics education research, we designed this study to specifically focus on physics students' understanding of complex number algebra, in a mathematical context that requires algebraic and geometric reasoning, but without knowledge of relevant physics.

In this paper, we illustrate middle-division physics students' difficulties and successful strategies when approaching a complex algebra problem. In particular, we aim to answer our primary research question: What facilities, difficulties, and strategies are exhibited by middledivision physics students in a simple complex number algebra problem that requires algebraic and geometric reasoning? Our analysis suggests that one characteristic of expertise in complex algebra is the ability to efficiently isolate approaches while reasoning with algebraic and geometric representations.

In Sec. II, we provide specific definitions of forms and representations of complex numbers and elaborate on the results of prior research. Section III includes a description of the interview prompt, the student participants, and the development of the coding scheme. Four particular sequences of steps, called approaches, that produce answers to the interview prompt are detailed in Sec. IV. We present results from students' answers to the prompt in Sec. V. Finally, we summarize and discuss results and their implications for instruction in Sec. VI.

\section{THEORETICAL BACKGROUND}

Our theoretical grounding develops from mathematics education research studies pertaining to mathematical reasoning and complex algebra. In this section, we introduce terminology and results from previous work that establishes that complex algebra fluency is hindered by the compartmentalization of representation [8] and preference for a form $[5,9,18]$.

\section{A. Coordinating forms and representations of complex numbers}

In this section, we describe the definitions of forms and representations of complex numbers that we refer to throughout this paper. Two central findings in previous literature contributed to the design of our study and to the discussion of our analyses: compartmentalization of representation and preference for a form.

The definitions of forms (rectangular, polar, and exponential) and representations (algebraic and geometric) of complex numbers are summarized in Table I, a modified version of those used by Karakok et al. [9].

A complex number is expressed using either an algebraic or geometric representation. For example, the rectangular form is represented in two ways: (i) algebraically as $x+i y$ where $x$ and $y$ are real numbers and (ii) geometrically with $x$ as the distance along the real axis and $y$ as the distance along the imaginary axis. A complex number may be 
TABLE I. Algebraic and geometric representations of the complex number $z$ in rectangular, polar, and exponential forms.

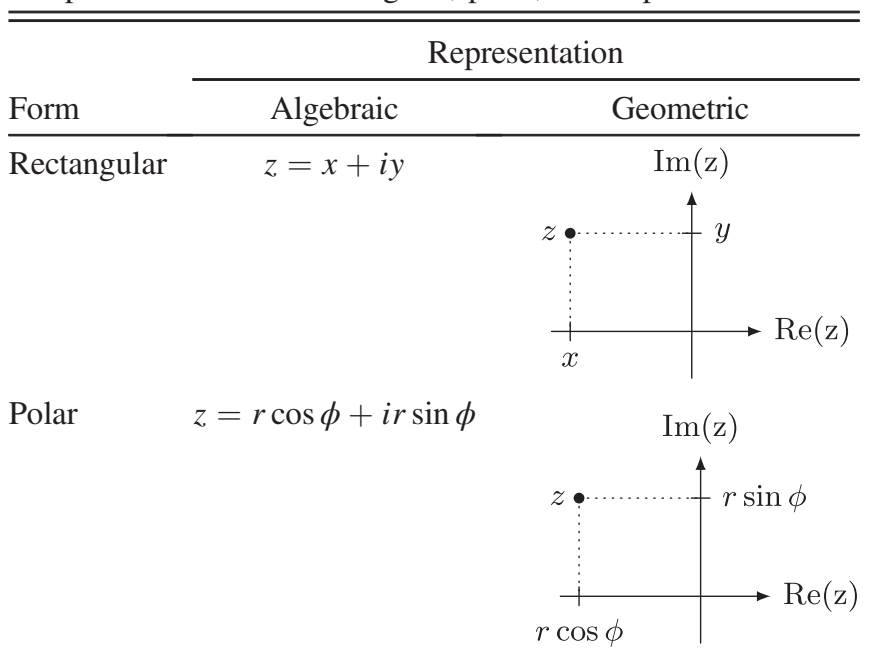

Exponential $\quad z=r e^{i \phi}$

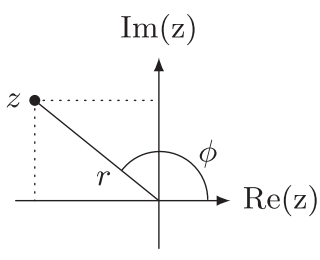

represented in either the rectangular or exponential form using either an algebraic or geometric representation. As shown in Table I, the polar form is a special case of the rectangular form and is listed as a distinct form; the polar form uses the parameters of the exponential form and serves as an intermediate between the two forms.

\section{Compartmentalization of representation}

Exclusive use of algebraic or geometric representations may be more practical to experts in certain physics contexts, but moving between algebraic and geometric representations is often necessary for solving physics problems. However, Browne found that middle-division physics students who were presented with a problem containing a particular representation tended to reason exclusively with that representation [19].

Compartmentalization of representation refers to the tendency for a person to reason with a single representation (a column in Table I). For example, a student may solve a complex algebra problem using only algebraic representations. Even though the student may switch between rectangular and exponential forms, a student's exclusive use of algebraic representations indicates their compartmentalization of the algebraic representation.

Panaoura et al. found that high school students tend to use either an algebraic or geometric approach while solving complex algebra problems [8]. One difficulty was the "phenomenon of compartmentalization" where some students were unable to convert geometric representations to corresponding algebraic representations, suggesting difficulties moving between representations of complex numbers. The high school students "considered the geometric and algebraic representations as two different and autonomous mathematical objects and not as two means of representing the same concept."

Soto-Johnson and Troup found two upper-division mathematics students did not exhibit full compartmentalization of representations [10]. When prompted for geometric reasons to explain the validity of algebraic statements, the students tended to reason first with algebraic expressions but shifted toward using both algebraic and geometric reasoning as they progressed $[10,11]$. The students exhibited mathematical sophistication using interchangeable algebraic and geometric reasoning.

A lack of compartmentalization of representation is indicative of connected conceptions of complex number representations. Simply, when students reason algebraically and geometrically, they do not compartmentalize representations. Experts do not compartmentalize representations. ${ }^{1}$

\section{Preference for a form}

A student may use both algebraic and geometric representations but work only with a single form (a row in Table I). A student who tends to work primarily with a single form exhibits a preference for that form.

Exhibiting preference for an inappropriate form can make problem solving more difficult or even impossible. Danenhower found that upper-division mathematics students demonstrated a preference for the rectangular form, often beyond the point of productivity and efficiency $[5,18]$. Most students delayed shifting to the exponential form, despite manipulations that were easier using that form. This behavior is exhibiting preference for the rectangular form.

Karakok et al. studied three mathematics teachers' understanding of the forms and representations of complex numbers after professional development sessions addressing complex number fluency [9]. The teachers displayed greater fluency working with the rectangular form than with the exponential form. Only the most experienced teacher demonstrated fluency moving between representations within each form. However, the teachers' tendency to work primarily with the rectangular form when solving complex number problems illustrates their preference for that form.

\footnotetext{
${ }^{1}$ An expert decision to use a single representation when a problem is efficiently solved using a single representation is not an example of compartmentalization of representation. Compartmentalization of representation inhibits a person's progress, understanding, and/or efficiency in solving a problem; it is not merely one's exclusive use of algebraic and/or geometric representations.
} 
We refer to the decision of working primarily with or using only representations of a single form as showing preference for the form. While compartmentalization of forms is conceivable, Danenhower's and Karakok et al.'s works suggest preference for a form to be more common among novices than complete compartmentalization.

A student who moves fluidly between both forms and representations (moves between both rows and columns in Table I) does not exhibit compartmentalization of a representation nor a preference for a form. In the context of complex algebra problems, these are expertlike behaviors.

\section{METHODS}

In this paper, we describe students' complex number fluency on a single interview task administered following an in-class introduction to complex algebra but prior to introducing physical applications. Using emergent coding techniques, we identified the approaches that students used to translate a geometric representation to several algebraic representations. To our knowledge and by the design of this study, the students had limited background in physical applications of complex number algebra but had experience with algebraic and geometric representations of complex numbers.

\section{A. Problem description}

The interview prompt, given in Fig. 1, uses a geometric representation of the complex number $z$ described by parameters in an equation, an angle, and a point on the imaginary axis. We designed the prompt to elicit common difficulties, previously identified through quizzes and exams $[17,20]$, that students encounter with complex algebra such as relating geometric and algebraic representations, choosing an appropriate form, and changing between forms. In part, we wanted to investigate the root of these common calculational difficulties. To do so, the interview prompt specifically targets how students handle angles that are not given from the positive real axis, how they interpret algebraic representations of the square of the norm, and how students change between and select forms and representations of complex numbers. By design, the prompt requires students to translate between algebraic and geometric representations, therefore students cannot compartmentalize representations. While students may exhibit preference for a form, they are unable to ignore either form.

Our previous work hinted that some middle-division physics students confuse aspects of triangle and circle trigonometry; students frequently made trigonometry errors while switching between forms [17]. We deliberately constructed the prompt to require students to distinguish between triangle and circle trigonometry. The angle $\alpha$ is provided from the negative imaginary axis to establish whether students recognize how to define an angle

$$
|z|^{2}=\ell^{2}
$$

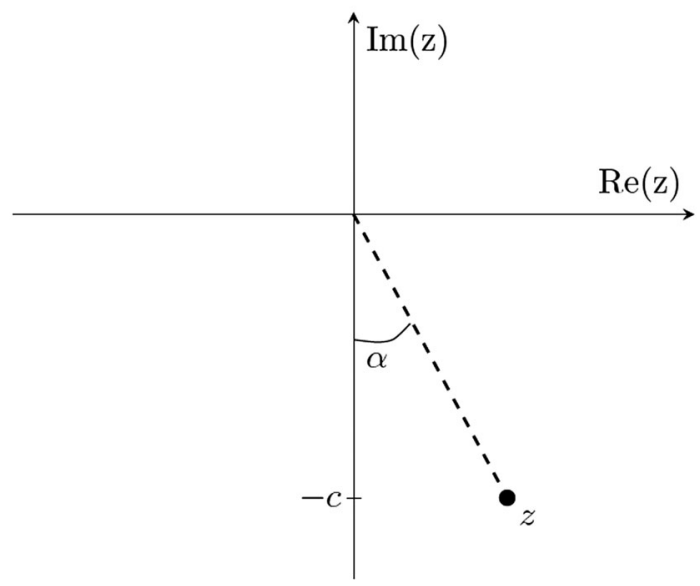

(a) Determine the rectangular and exponential forms of the complex number, $z$, in terms of $\ell$ and $c$.

(b) Determine the rectangular and exponential forms of the complex number, $z$, in terms of $\ell$ and $\alpha$.

FIG. 1. Interview prompt given to students in two parts as the third question in the protocol developed for Fall 2015. Part (a) was given immediately prior to and separately from part (b). Both parts were given using the same diagram and equation.

associated with the exponential form (i.e., from the positive real axis). The angle $\alpha$ cannot be directly incorporated in the exponential or polar forms; students must consider the angular displacement from the positive real axis, which requires students to distinguish between triangle and circle trigonometry. If students choose to directly use $\alpha$, the positioning of the complex number in the fourth quadrant requires that they reason about signs associated with the real and imaginary parts.

Distinguishing between the square $\left(z^{2}\right)$ and the square of the norm $\left(|z|^{2}\right)$ was a common calculational difficulty in students' written work [17], and we suspected this to be a difficulty among students who relied on algebraic reasoning with complex numbers. We incorporated the equation $|z|^{2}=\ell^{2}$ to provide information that the distance from the origin to $z$ is $\ell$. This step requires students to geometrically interpret an algebraic representation. All calculations in our previous work were prompted only through algebraic representations, so we created a situation that required algebraic and geometric reasoning about the norm to determine whether these calculational difficulties persisted when this reasoning was invoked.

In our previous research, we also identified that middledivision students had difficulties switching between and choosing appropriate forms of complex numbers in mathematics contexts [17]. The prompt requires students to provide both rectangular and exponential forms, which one could complete by independently generating forms or by switching between forms. 
After pilot interviews with two upper-division students, one graduate student, and an expert, we settled on the twopart, open-ended prompt to allow for many different solution paths. We selected combinations of parameters in parts (a) and (b) because there exist efficient and preferable paths in determining the algebraic representations of $z$, as discussed in Sec. IV. Our intention with the order of parts (a) and (b) was to gauge whether students exhibited sustained preference for a form, which Danenhower found was prevalent in upper-division mathematics students' solutions [5]. Part (a) is easiest to first solve in rectangular form and part (b) in exponential or polar forms.

The parts, given in Fig. 1, were asked in succession, with the students working through part (a) prior to the interviewer (E. M. S.) introducing part (b). Each question began with the interviewer reading aloud the prompt and providing students with a printed copy of the prompt, diagram, and equation. We used a think-aloud, semistructured protocol to encourage students to discuss their process throughout the interview. The interviewer prompted students to explain their reasoning throughout the problem. Additionally, the interviewer asked some questions intended to redirect students' attention to the prompt. In one instance the interviewer explicitly provided a student with information regarding translation of the geometry to an algebraic representation. All interviews were video recorded using two cameras to capture both the board work and gestures of the students.

\section{B. Background of students}

We conducted interviews during week 5 of the junior year. At OSU most of the junior-level courses-called Paradigms - revolve around concepts (e.g., energy, symmetry, eigenstates) underlying the traditional physics subdisciplines [21]. From comparisons with curricula at other institutions, the students had comparable physics and mathematics backgrounds to other physics students at the beginning of middle-division courses.

The instructor designed and implemented an introduction to complex algebra with an emphasis on changing between forms and representations during the second and third days of the Paradigms curriculum in Fall 2015. Students were introduced to algebraic and geometric representations as given in Table I. For example, students were introduced to multiplication by a phase as a rotation in the complex plane. We developed supplemental homework problems to provide students with practice performing complex algebra manipulations. We designed and administered announced assessments on complex algebra throughout weeks 1-3. The interviews were conducted prior to week 7 , when physical applications of complex numbers and functions are first introduced in the context of harmonic oscillators.

We selected seven students to participate in the interview based on performance on complex algebra assessments, mathematics and physics backgrounds, declared major, and availability during week 5. Our selection of students represents the diversity of backgrounds and mathematics experiences of students entering the Paradigms curriculum. All students stated they intended to be physics majors and had completed a year-long sequence of introductory physics. Four students (C, E, F, and G) were concurrently enrolled in a modern physics course, and the others (A, B, D) completed the course during Spring 2015. At least two students (B and F) completed relevant mathematics courses at community colleges and transferred to OSU either prior to or at the beginning of the junior year. All students interviewed were male; more than $90 \%$ of the students entering the Paradigms curriculum in Fall 2015 were male.

Prior to the in-class introduction to complex algebra, five students (B, C, E, F, and G) self-reported having had "one or two days" of previous formal instruction on complex numbers and/or algebra, and two students (A, D) selfreported "more extensive" experience. Students reported having experience with complex algebra in high school (A, E, G), college trigonometry (B, F), differential equations (C, E, G), and sequences and series (B, F) courses. Student D completed a complex analysis course in mathematics, and student $\mathrm{C}$ indicated self-study of complex algebra topics.

\section{Identifying codes and descriptions of codes}

All interviews were conducted and all videos were transcribed prior to beginning the coding process. We used

TABLE II. Summary of codes for translations of geometry in the prompt to algebraic representations. Italicized codes are specific errors in executing a step.

Fundamental algebraic and geometric relationships

Angle

X1. Student defines the angle geometrically from the positive real axis

a. Student geometrically identifies $\phi$ and $\alpha$ as the same angle

X2. Student determines a correct algebraic expression for $\phi$ $(\phi=\alpha+3 \pi / 2+2 \pi n)$

a. Student sets $\alpha=\phi$

b. Student finds an incorrect algebraic expression for $\phi$ (except for $\phi=\alpha)$

Norm Squared

Y1. Student finds that $\ell$ is distance from the origin to $z$ a. Student questions the geometric interpretation of $|z|^{2}=\ell^{2}$

Y2. Student performs a calculation involving $|z|^{2}$ a. Student confuses calculations of $|z|$ and $|z|^{2}$

Imaginary Part

Z1. Student recognizes $-c$ is the imaginary part of $z$ from the geometry

a. Student claims the imaginary part of $z$ is $c$

b. Student sets $-i y=-c$ or $i y=c$ 
TABLE III. Summary of codes describing approaches. Italicized codes are specific errors in executing a step. The top approaches rely on triangle geometry. The bottom approaches rely on polar geometry.

\begin{tabular}{|c|c|c|}
\hline & & Triangle trigonometry approach \\
\hline \multirow[t]{8}{*}{ Triangle geometry } & $\mathrm{T} 1$. & Student identifies right triangle in order to apply SOH CAH TOA \\
\hline & $\mathrm{T} 2$. & $\begin{array}{l}\text { Student maps the problem's parameters to } \mathrm{SOH} \text { CAH TOA } \\
\text { a. Student uses a negative length }\left(\text { e.g., } \cos \alpha=\frac{-c}{\ell}\right) \\
\text { b. Student selects the sides of the triangle incorrectly }(\text { e.g., } \cos \alpha=\text { adj/hyp }=\ell / c)\end{array}$ \\
\hline & $\mathrm{T} 3$. & Student solves for the unknown part of the trigonometric expression) \\
\hline & T4. & Student applies appropriate signs for the quadrant associated with $z$ \\
\hline & & Pythagorean theorem approach \\
\hline & P1. & Student recognizes triangle can be used with the Pythagorean theorem \\
\hline & $\mathrm{P} 2$. & Student maps the problem's parameters to the Pythagorean theorem \\
\hline & P3. & Student solves for the unknown side of the triangle \\
\hline \multirow[t]{8}{*}{ Polar geometry } & & Circle trigonometry approach \\
\hline & $\mathrm{C} 1$. & Student recognizes the real part corresponds with the cosine and the imaginary part with the sine \\
\hline & $\mathrm{C} 2$. & $\begin{array}{l}\text { Student uses algebraic expressions of the angle and/or magnitude to express a form } \\
\text { a. Student uses an incorrect definition of the form }\end{array}$ \\
\hline & C3. & $\begin{array}{l}\text { Student uses a known relationship between the real and imaginary parts of a complex number used } \\
\text { (e.g., } \phi=\arctan (y / x)\end{array}$ \\
\hline & & a. Student uses incorrect relationships between forms and parameters $[$ e.g., $\phi=\arctan (|y| /|x|)]$ \\
\hline & & Norm squared approach \\
\hline & N1. & $\begin{array}{l}\text { Student maps the problem's parameters to a calculation of the norm squared } \\
\text { a. Student incorrectly squares the imaginary part of the complex number }\end{array}$ \\
\hline & $\mathrm{N} 2$. & $\begin{array}{l}\text { Student solves for the unknown part of } z \\
\text { a. Student solves for the known part of } z\end{array}$ \\
\hline
\end{tabular}

emergent coding of the transcripts to identify the steps that students used to solve the task. In the first round of the analysis, we identified fine grain-size categorizations of individual students' responses to the prompt using open coding techniques. Our subsequent coding narrowed the scheme based on similarities among the codes developed for individuals' solutions using constant comparative analysis methods [22]. Each code corresponds to a step in a solution to the prompt. In the final versions of the

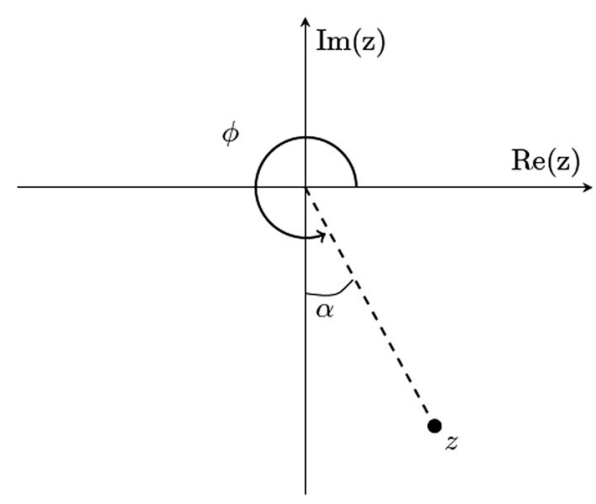

FIG. 2. The angle $\phi$ is defined to be counterclockwise from the positive real axis and is associated with a circle trigonometry approach. The angle $\alpha$ is provided in the interview prompt as the angle from the negative imaginary axis and is used in the triangle trigonometry approach. coding scheme, the codes were categorized into sequences of steps that result in correct algebraic representations of the complex number; we refer to these particular sequences of steps as approaches. We identified incorrect steps in students' solutions during the initial stages of coding but did not develop or apply codes to these incorrect statements until the scheme was nearly final.

The final coding scheme, given in Tables II and III, was applied to students' responses, which are summarized by codes in the Appendix, Fig. 3. In Sec. V, we describe results that emerged from the data.

Students may correctly solve the prompt in many ways, and the codes encompass significant steps made by students. Table II contains the steps involving the translation of the geometric information provided in the prompt into algebraic statements. Table III contains the general steps of four approaches that students used while solving parts (a) and (b): triangle trigonometry, Pythagorean theorem, circle trigonometry, and norm squared. In this paper, we focus primarily on the approaches (Table III), but we also include the codes corresponding to students' initial steps in the problem (Table II) because they are foundational to the approaches.

Obtaining particular combinations of the parameters in an algebraic representation is expedited by selecting an efficient approach. The Pythagorean theorem and norm squared approaches are most useful for part (a) in obtaining the rectangular form in terms of $\ell$ and $c$. The circle 
trigonometry approach is most useful in part (b) for constructing both the exponential and polar forms in terms of $\ell$ and $\alpha$.

\section{DESCRIPTIONS OF APPROACHES}

In this section, we describe sequences of steps that produce solutions to the prompt to explain the four approaches. The following descriptions are of each approach, without extraneous steps, and the resulting algebraic representation(s).

\section{A. Triangle trigonometry}

An ideal triangle trigonometry approach begins with recognizing the right triangle containing the angle $\alpha$ (T1). The hypotenuse of the triangle is found to be $\ell$ by using the geometric interpretation of $|z|^{2}=\ell^{2}$ (Y1). The given magnitude of the imaginary part of the complex number $c$ provides the height of the triangle (Z1). Trigonometric relationships relating $c, \ell$, and $\alpha$ determine the magnitudes of the real and imaginary parts of $z$, such as $|x|=\ell \sin \alpha$ and $|y|=\ell \cos \alpha$ (T2 and T3). Finally, the signs are selected from the quadrant; in this case, the imaginary part is negative, and the real part is positive (T4). Using a triangle trigonometry approach, the rectangular form of the complex number may be expressed as $z=\ell \sin \alpha-$ if $\cos \alpha$ or $z=c \tan \alpha-i c$.

\section{B. Pythagorean theorem}

The same triangle and initial steps are used with the ideal Pythagorean theorem approach (P1). These include finding the hypotenuse and height of the triangle with the information provided by the prompt (P2). The magnitude of the real part of the complex number is found (P3) by applying the Pythagorean theorem (P2). With this approach, the rectangular form is expressed as $z=\sqrt{\ell^{2}-c^{2}}-i c$.

\section{Circle trigonometry}

An ideal circle trigonometry approach uses a positive angle, as in Fig. 2, defined counterclockwise from the positive real axis, $\phi(\mathrm{X} 1$ and $\mathrm{X} 2)$, and $\ell$, the distance from the origin to $z$ is found from the geometric interpretation of the relation $|z|^{2}=\ell^{2}$ (Y1). Using these algebraic expressions, the exponential form is expressed as a magnitude multiplied by a phase (C2). The polar form uses the association of the real part with the cosine of the angle and the imaginary part with the sine $(\mathrm{C} 1)$ - an analogue to the use of polar coordinates. Algebraic representations resulting from the circle trigonometry approach include the exponential form $z=\ell e^{i(\alpha+3 \pi / 2)}$ and the polar form $z=\ell \cos (\alpha+3 \pi / 2)+i \ell \sin (\alpha+3 \pi / 2)$. Clockwise angles expressed negatively (e.g., $\phi=-\pi / 2+\alpha$ ) are contained within the circle trigonometry approach.

\section{Norm squared}

Finally, the ideal norm squared approach is where an algebraic calculation of the square of the norm of $z$ is used to determine the real part. The calculation of the square of the norm is performed: $|z|^{2}=z z^{*}=(x+i y)(x-i y)=$ $x^{2}+y^{2}$ (Y2). The imaginary part, $y$, is known from the prompt to be $-c(\mathrm{Z} 1)$, and the equation in the prompt provides $|z|^{2}=\ell^{2}$. These equations are used to solve for the real part of the complex number ( $\mathrm{N} 1$ and $\mathrm{N} 2$ ). The algebraic representation resulting from this approach

TABLE IV. Example of the coding for a portion of student A's interview during part (a). The excerpt corresponds to the first two upper boxes in Fig. 3 for student A.

\begin{tabular}{|c|c|c|}
\hline Codes & & Transcript \\
\hline \multirow[t]{5}{*}{ Triangle Trigonometry } & $\mathrm{Z1}$ & $\begin{array}{l}\text { A: Um, the distance down is minus } c \text { [draws dotted line from origin to point, labels }-c \text { on imaginary } \\
\text { axis], } z \text { [labels point } z] \text {, alpha [labels } \alpha \text { ]. So to find, so the imaginary component, uh, is minus } i \\
c \text { [writes }-i c] \text {, }\end{array}$ \\
\hline & $\mathrm{T} 1$ & the real component is going to be related to the imaginary component by this angle alpha. \\
\hline & $\mathrm{T} 2$ & $\begin{array}{l}\text { Um, so the, real component, we can call it } a \text { [labels real component on diagram as } a \text { ], um, } \\
\text { tangent of alpha is equal to, uh, } a \text { over } c \text { [writes } \tan \alpha=a / c] \text {, yeah, yeah that's right. }\end{array}$ \\
\hline & $\mathrm{T} 3$ & Um, $a$ equals $c$ tan alpha [writes $a=c \tan \alpha$ ]. \\
\hline & & $\begin{array}{l}\text { So this imaginary number in, in terms of } c \text { is } c \tan \text { alpha minus } i \text { [writes } z \text { as } c \tan \alpha-i c \text { ]. Um, } \\
\text { now in, okay so that's rectangular [underlines } c \tan \alpha-i c \text { ]. }\end{array}$ \\
\hline \multirow[t]{5}{*}{ Circle Trigonometry } & Y1 & $\begin{array}{l}\text { And [pause to read prompt], um, oh } z \text { squared, okay so magnitude of } z \text {, squared equals } \ell \\
\text { squared, so then this distance here [draws line from origin to } z \text { ] is } \ell \text { [labels line as } \ell \text { ]. }\end{array}$ \\
\hline & $\mathrm{T} 1$ & $\begin{array}{l}\text { So in that case, uh, the imaginary and real components can be represented with, uh, sines and } \\
\text { cosines of this angle alpha. }\end{array}$ \\
\hline & $\mathrm{X} 1$ & And we write, just for consistency [draws angle from positive real axis to $z$ ] \\
\hline & $\mathrm{X} 2$ & to make this whole angle, uh, three pi over two plus alpha [writes $3 \pi / 2+\alpha$ ]. \\
\hline & $\mathrm{C} 1(\& \mathrm{C} 2)$ & $\begin{array}{l}\text { So the real component is, uh, cosine is } \ell \text { cosine of three pi over two plus alpha, I believe [writes } \\
c \tan \alpha-i c=\ell \cos (3 \pi / 2+\alpha) \text { ], yes. }\end{array}$ \\
\hline
\end{tabular}


is the same as the Pythagorean theorem approach: $z=\sqrt{\ell^{2}-c^{2}}-i c$. Though resulting in the same algebraic representation as the Pythagorean theorem approach, the norm squared approach is driven by an algebraic calculation of the square of the norm. The Pythagorean theorem approach is driven by a student's discussion of a right triangle to generate an algebraic relationship between the sides of the triangle rather than a calculation of the square of the norm.

\section{E. Example of transcript coding}

As an illustration of the coding scheme applied to a transcript, Table IV provides an excerpt where student A separately used the triangle and circle trigonometry approaches. He initiated the solution by determining the rectangular form using a triangle trigonometry approach. He then used a circle trigonometry approach to determine the real part of the polar form. Following further exchanges with the interviewer, not shown in Table IV, student A correctly constructed the polar form in terms of $\ell$ and $\alpha$.

\section{RESULTS}

\section{A. Initial steps and unique sequences of steps}

Each student engaged in steps associated with multiple approaches, but individuals' sequences of steps were unique after their initial steps. None of the students followed an "ideal" sequence of steps such as beginning part (a) with either the Pythagorean theorem or norm squared approach to find the rectangular form. This led all students to developing extraneous algebraic representations, which were not direct responses to the prompt.

Students coordinated sequences of steps associated with particular approaches at various stages within their responses, and there was no consistency among different students' sequences of steps throughout the problem. These unique sequences of steps contributed to a variety of algebraic representations of the complex number in students' solutions.

As the first step, students must translate at least some information provided in the prompt into algebraic expressions; the possible first steps are given in Table II. Different students first engaged with different steps (see Fig. 3 in the Appendix). Students B, C, D, F, and G used $|z|^{2}=\ell^{2}$ to discuss the geometric (Y1) and/or calculational aspects (Y2) of the norm and the square of the norm. Students B, C, and $\mathrm{E}$ began with the geometry of the angle $\phi(\mathrm{X} 1$ and $\mathrm{X} 2)$. Student A started by using the diagram to identify the imaginary part of the complex number (Z1).

Presumably, some students had a specific goal from the outset of the problem, influencing their decisions during the first steps. For example, student A identified the imaginary part of $z$, given in Table IV, which led to a successful and efficient triangle trigonometry approach.
After obtaining the rectangular form, he used the geometry of $|z|^{2}=\ell^{2}$ and the evaluation of the angle associated with the complex number to determine the polar form. These specific combinations of initial steps to develop algebraic representations lend themselves to the approach he used. This suggests that student A planned ahead and intentionally selected the initial steps. We suspect students $\mathrm{C}$ and $\mathrm{F}$ proceeded with or quickly determined similar plans.

The initial steps of students B, D, E, and G, on the other hand, did not immediately lend themselves to their chosen approaches, suggesting less planning and overall strategy in solving. For example, when solving part (a), student D used steps from several approaches, perhaps his initial steps did not clarify a path to determine an algebraic representation. He began with calculations of the square of the norm (finding $|z|=\sqrt{x^{2}+y^{2}}$ by multiplying $z z^{*}$ for $z=x+i y$ ) and continued to reference the calculation without connection to the norm squared approach:

D: Um, so this squared, $\ell$ squared [writes $|z|^{2}=\ell^{2}$ ], we're just saying that magnitude of $z$ is equal to $\ell$ [writes $|z|=\ell$ ], which makes sense. Y2 Um, but again we don't know what $x$ and $y$ are, but we have an angle and we have a length, um, or we have a position $c$. We don't really have a length though. But let's just try it, so what could this be [draws dotted line from -c to z]? Ummm

ES: What were you thinking about doing?

D: Yeah, what was I thinking about, I'm trying to, um, I'm trying to find an $x$ length and a y length that I can relate here [pointing at $\sqrt{x^{2}+y^{2}}$ ] . N1 And then if we want to express this in, um, I guess my thought process is, if I can find, if I can say that $x$ is equal to cosine theta [writes $x=\cos \theta$ ] and $y$ is equal to, sine theta [writes $y=\sin \theta$ ]. Then I also need, um, I need an [changes to $x=|z| \cos \theta$ and $y=|z| \sin \theta]$, we can read that.

Despite beginning with a calculation of the square of the norm-which suggests his intention to use the norm squared approach-student D demonstrated little knowledge of the usefulness of this particular first step. He did not connect the algebraic expression of the magnitude of $z$ to also represent the geometric distance from the origin to $z$ and turned to the position on the imaginary axis to find a relevant length. Interestingly, he used language describing $c$ as a position rather than a length, which suggests that he was attempting to use geometric reasoning but did not attempt to geometrically reason with the algebraic expression of the magnitude of $z$. He proceeded through the problem using various approaches but did not follow through with the norm squared approach. This suggests the initial step of calculating the square of the norm was exploratory rather than a predetermined step toward an 
explicit goal. Students B, E, and G also did not immediately use their initial steps in an approach, contrasting with students $\mathrm{A}, \mathrm{C}$, and $\mathrm{F}$ who did.

\section{B. Isolation of approach}

In this section, we use the term isolation of approach when a student uses steps associated with a single approach (triangle trigonometry, Pythagorean theorem, circle trigonometry, or norm squared) that results in an algebraic representation. A student may isolate several approaches while responding to the prompt. Students who consistently isolate approaches tend to obtain correct algebraic representations of the complex number. To illustrate, each box in Fig. 3 (found in the Appendix) represents an episode of problem solving when neither the student nor interviewer mentioned what the prompt asked; Ref. [20] further describes the features represented by the boxes. When an approach is isolated, a box contains codes corresponding to a single approach.

Isolation of approach, a productive problem solving strategy in this situation, is different from the idea of compartmentalization of representation, described as a difficulty by Panaoura et al. [8]. Compartmentalization of representation may be observed in only the precursory steps to an approach, the codes contained in Table II. However, each approach requires students to translate the geometric representation to algebraic statements. Algebraic and geometric representations cannot be compartmentalized within an approach; students must engage in algebraic and geometric reasoning.

While most students isolated approaches during portions of the interview, students $\mathrm{A}$ and $\mathrm{F}$ isolated approaches consistently and effectively while working through the task. In each instance of using a particular approach, student A was especially successful at avoiding extraneous exploration of the problem and committed to his chosen strategy. His consistent follow through with each approach resulted in correct algebraic representations in rapid succession. He took clear and efficient sequences of steps towards algebraic representations, which suggests that he was able to isolate approaches.

Student E failed to isolate an approach in several instances. He often did not follow through with an approach prior to bringing in another. For example, he began with a triangle trigonometry approach but interrupted with the Pythagorean theorem approach:

E: Um, I have an adjacent side and, alright so I could do the cosine portion which would uh, which would be our, c, um, cosine alpha, alpha wow, alpha [writes $\langle c \cos \alpha]$, comma, um, this is going to be our adjacent leg, um, and now, what I want to write is that this, this portion of the leg which would be, $\quad \mathbf{T 2 b} \& \mathbf{T 3}$ $u$, we could get that from Pythagorean theorem so $\ell$ squared, uh, equals, um,

P1 c squared plus, um, let's call it a side squared [writes $\left.\ell^{2}=c^{2}+s^{2}\right]$.

That's one way, but there's an easier way to do this but kind of like blanking.

ES: It happens!

E: Okay, uh, so um, s squared equals $\ell$ squared minus $c$ squared [writes $s^{2}=\ell^{2}-c^{2}$ ], so $s$ equals the square root of $\ell$ squared minus $c$ squared [writes $s=\sqrt{\ell^{2}-c^{2}}$ ]. Right, um, [inaudible] so um, so we know that this side $s$ [indicating distance from $-c$ to $z$ ] is equal to this [indicating $s=\sqrt{\ell^{2}-c^{2}}$ ]. So square root of $\ell$ squared $c$ squared sine alpha [writes $\left\langle c \cos \alpha,-\sqrt{\ell^{2}-c^{2}} \sin \alpha\right\rangle$ ]. And I put a minus sign to correct for the, it's gonna be the negative imaginary direction.

T4

The resulting algebraic representation, $z=\langle c \cos \alpha$, $\left.-\sqrt{\ell^{2}-c^{2}} \sin \alpha\right\rangle$, inappropriately blended the two approaches. Student E demonstrated sufficient knowledge to use the Pythagorean theorem approach, however, his blended use of the triangle trigonometry approach resulted in mangling together the two approaches. Not only did his lack of isolation lead him to inefficient solutions but also contributed to his inability to recognize progress within the task.

Student D also exhibited a tendency to not isolate an approach. In one portion of Part (a), he brought together three distinct approaches-norm squared, circle trigonometry, and triangle trigonometry-while attempting to map the problem's parameters to the rectangular form. He was able to demonstrate understanding of several steps within multiple approaches, however, his struggle to isolate an approach caused him to deviate several times from an efficient sequence of steps. Student D recognized his extended process and commented: "I feel like I'm going really slow" and "I feel like I'm going all over the place." These statements suggest he was actively monitoring his problem-solving process and was aware of his struggle to coordinate an efficient approach. After refocusing on a single goal, student $\mathrm{D}$ shifted to the triangle trigonometry approach, which he attempted to isolate. He was more successful isolating an approach after his self-reflective comments. However, he nevertheless made several errors, resulting in an incorrect algebraic representation.

\section{Blended use of circle and triangle trigonometry approaches}

The selection and execution of trigonometry techniques were the most commonly occurring issues; five out of the seven students made various trigonometry related errors. Four of the five students' trigonometry issues arose from blended use of triangle and circle trigonometry 
approaches. Three of the seven students (students B, C, and $\mathrm{E}$ ) blended triangle and circle trigonometry steps, shown in Fig. 3 in the Appendix by codes enclosed by red boxes, which are separately correct but when used together lead to incorrect conclusions. Student D switched between and inappropriately combined the approaches in several segments of his solutions, but his individual steps are distinguishable.

Students B and E used-from circle trigonometrythat the cosine is associated with the real part and the sine with the imaginary part $(\mathrm{C} 1)$ and-from triangle trigonometry-that signs are assigned according to the quadrant (T4). This may be an artifact of not distinguishing between the angle (see Fig. 2) associated with each approach: $\phi$ is used with circle trigonometry and $\alpha$ with triangle trigonometry.

Student B decided to relabel the angle $\alpha$ as $\phi$ and caused confusion for both himself and the interviewer by conflating angle definitions from both triangle and circle trigonometry. He recalled the imaginary part is associated with $\sin \phi$ and the real part with $\cos \phi(\mathrm{C} 1)$-from circle trigonometry. Student B overlayed this with signs from triangle trigonometry by accounting for the quadrant of the real and imaginary parts (T4). This resulted in a template, $\cos \phi-i \sin \phi$, which he used throughout part (a) and pulled into part (b). He merged distinct trigonometric approaches, causing persistent issues. Students D and E also made errors associated with the definition of the angle, suggesting their blended use of the triangle and circle trigonometry approaches.

Additionally, students exhibited difficulties isolating a triangle or circle trigonometry approach when mapping expressions from triangle trigonometry to a relationship that relies on circle trigonometry, and vice versa. For example, student $\mathrm{C}$ identified the triangle containing $\alpha$ on the diagram and determined that the tangent of $\alpha$ is related to the real (he labeled as " $a$ ") and imaginary (labeled " $b$ ") parts:

C: Um, this would be, some a, some b [labels real part as $a$ and imaginary part as $b$ on the diagram]. Then I want, um, $b$, yeah the tan of alpha is going to be a over $b$ [writes $\tan \alpha=a / b$ ].

T1

So that means that, actually we're given $b$, never mind. I won't erase that. So this $b$ [indicating $b$ on diagram] is actually equal to negative $c$ [writes $-c=b$ ]. $\quad \mathbf{Z 1}$ So tan of alpha is gonna be a over negative $c$ [writes $\tan \alpha=a /-c]$.

T2a

So a is gonna be equal to negative $c$ tan alpha [writes $a=-c \tan \alpha]$.

T3

So now if we want to write in terms of a plus $b i$ [writes $a+b i]$, that means that a is going to be the, a has to be positive, yeah tan of alpha, I need to choose my angle right. Because I have to make this spit out a positive [indicating $a=-c \tan \alpha$ ] version because a is positive.
The tangent of the angle $\phi$ is defined to be the ratio of the imaginary and real parts of a complex number from circle trigonometry $(\tan \phi=y / x)$. Student $\mathrm{C}$ used the triangle containing $\alpha$ to determine the tangent in terms of the lengths of the triangle's sides. However, he then used the real and imaginary parts of the complex number in the tangent relationship. In doing so, he pulled from the circle trigonometry approach by using the negative length, $-c$, because it is the imaginary part of the complex number.

Blended use of the triangle and circle trigonometry approaches results in an incorrect expression for the real part of the complex number. Student $\mathrm{C}$ demonstrated understanding of the geometry and appropriate signs through his statements that the real part, $a$, must be positive. The algebraic expression $a=-c \tan \alpha$, however, is a negative number. The use of symbols, rather than numbers, may conceal the signs and affect his ability to assess the appropriateness of the resulting algebraic expression. As the student progressed in the task, he continued to use the result by directly blending triangle and circle trigonometry approaches:

$C$ : Tan of alpha is gonna be a over $b$, so that means that the alpha is actually going to be the inverse tangent of $a$ over $b$ [writes $\alpha=\tan ^{-1}(a / b)$ ].

T3/C3

\section{Preference for forms}

Interestingly, some students (B, D, G) who did not consistently isolate approaches may have been exhibiting a preference for the rectangular form, suggesting an avenue to investigate is whether preference for a form inhibits isolation of approaches. In most cases, students began with the objective of finding the rectangular form, evident by their choices of triangle trigonometry or Pythagorean theorem approaches. While part (a) was designed so that students would begin with the rectangular form, part (b) was developed to examine whether students would start from the exponential form. Students B, D, F, and G chose to work toward the exponential form only at the conclusion of both parts (a) and (b), which suggests their preference for the rectangular form. Every student engaged with the explicit goal of finding an algebraic representation of the exponential form, at least briefly, however, sometimes this only occurred when the interviewer encouraged the student to do so. Only student $G$ failed to obtain an algebraic representation in the exponential form, which suggests that changing between forms remains a difficulty for some students. Most students (B, D, E, F) obtained incorrect algebraic representations of the complex number in the exponential form but not usually resulting from difficulties changing between rectangular and exponential forms.

An alternative explanation for students' common choice to first use the rectangular form is the listed order in the 
prompt: rectangular precedes exponential. However, advanced undergraduate and graduate students did not have an observable preference for a particular form during pilot interviews. Additionally, from analysis of students' quizzes and exam solutions, some students consistently chose to work with the rectangular form, even when the exponential form was more efficient $[17,20]$. This suggests that students B, D, F, and G exhibited novicelike preference for the rectangular form, which may indicate that their abilities to select appropriate forms in simple situations are still developing.

\section{DISCUSSION AND IMPLICATIONS}

In this paper, we describe students' fluency in translating between geometric and algebraic representations of a complex number. Our primary finding presented in this paper has not been identified in previous studies: Success in complex algebra tasks requires students to isolate approaches. Students who isolated approaches-triangle trigonometry, circle trigonometry, Pythagorean theorem, and norm squared-tended to be more successful in determining corresponding algebraic representations than those who did not.

\section{A. Isolating approaches}

While students A and F consistently isolated approaches throughout the interview, most students struggled to do so. In particular, student $\mathrm{D}$ incorporated many extraneous steps into his solution but recognized his inability to isolate a single approach, which suggests his monitoring of his problem solving process. In at least four students' responses $(\mathrm{B}, \mathrm{C}, \mathrm{D}$, and $\mathrm{E})$, incorrect algebraic representations resulted from students' blended use of the triangle and circle trigonometry approaches, suggesting that distinguishing between triangle and circle trigonometry is a difficulty for some middle-division physics students.

Expert fluency is marked by moving between and selecting forms and representations effectively within a context. In complex algebra, experts need to not only engage in algebraic and geometric reasoning but to also choose and isolate an appropriate approach to the problem. While compartmentalization of representations may be common to novices' problem solving, our study design required students to use algebraic and geometric representations from the outset of the problem. This allowed us to identify isolating an approach as an additional feature of expertlike fluency in complex algebra.

Reasoning with both algebraic and geometric representations is needed in many physics contexts. However, experts must also be able to recognize the important features of a problem to select a particular solution path without distraction by extraneous features. These complementary strategies may assist experts in selecting appropriate forms to efficiently work through a problem. We encourage additional exploration of the role of isolating approaches in physical contexts.

\section{B. Difficulties isolating approaches: Trigonometry}

Distinguishing between and executing triangle and circle trigonometry approaches were the most prominent difficulties for students. Most students encountered some difficulties with the translation of the geometric representation to an algebraic representation. Only student A performed every step correctly and completed both Parts (a) and (b) without an error, however, five of the seven students (B, C, D, E, F) made errors related to trigonometry. This suggests difficulties with trigonometry persist into middle-division physics courses but may manifest primarily in distinguishing between circle and triangle trigonometry. Encouragingly, all students engaged in translating between algebraic and geometric representations, suggesting their developing facility with algebraic and geometric reasoning in a simple complex algebra context.

Even while isolating approaches, some students incorporated extraneous trigonometry steps. For example, student A referred to the right triangle containing $\alpha$ prior to using a circle trigonometry approach. We maintain that student A isolated the circle trigonometry approach because he used the angle $\alpha$ as a way to recognize that he needed the angle to be defined counterclockwise from the positive real axis to map parameters to the polar form. This requires connected conceptions of the algebraic and geometric representations of a complex number because the geometric representation evokes a clear path to determining an algebraic representation.

The triangle and circle trigonometry difficulties that we identified in complex algebra contexts may be related to those previously found in introductory physics. Mikula and Heckler found that four populations of introductory physics students struggled with trigonometry associated with vector decomposition, such as confusing trigonometric functions with certain angle configurations [23]. Ozimek et al. found that algebra-based physics students struggled to retain and transfer circle trigonometry from math into physics [24]. Instructional emphasis on triangle trigonometry in introductory physics courses may contribute to middle-division students' preference for triangle trigonometry approaches or difficulties distinguishing between triangle and circle approaches.

\section{Limitations of the coding scheme}

We developed the coding scheme from the steps that students executed as they completed the problem. However, explanations for why they chose to execute particular steps or sequences of steps is not captured by the coding scheme. For example, in Sec. VA, student D's response includes a step associated with the norm 
squared approach because he begins with an explicit calculation of the square of the norm. He then begins to develop algebraic representations to plug into his calculated expression of the norm. However, his language (e.g., " $x$ length") hints that his explanations of his steps may have involved a triangle, which is not captured by coding his executed steps. The analyses presented here focus on students' steps and order of those steps but do not capture explanations of students' chosen sequence of steps.

\section{Implications for classroom instruction}

Students transitioning from lower- to upper-division physics courses may lack extensive experience with complex algebra. Because complex algebra is pervasive throughout upper-division physics, students require fluency in calculations, which often require both algebraic and geometric reasoning. Instruction focused on the geometric relationships, specifically relating algebraic and geometric representations, may assist in students' development of complex algebra skills.

All of the students interviewed retained some of the information from the in-class introduction to complex algebra, which occurred five weeks prior to the interview. Yet some students encountered difficulties geometrically defining forms of a complex number despite explicit and extended instruction and corresponding assessment. Results from postinstruction quizzes suggest that similar difficulties arise with numerical, not just symbolic, representations [20]. From interviews conducted with different students late in the junior year at OSU (unpublished), we suspect that sophisticated coordination of algebraic and geometric representations of complex functions develops gradually throughout middle-division physics courses.

Increasingly sophisticated complex algebra is necessary as physical systems are introduced in upper-division courses. Students' complex algebra development and extension to physical applications may be aided by instruction focused on the relationships between algebraic and geometric representations by drawing out mathematical features that are commonly exploited in physics problem solving. Complex algebra instruction immediately prior to applications may provide an opportunity for students to usefully connect geometric and algebraic (and perhaps, physical) representations.

In lower- and middle-division physics courses, incorporating instructional emphasis on the distinction between triangle and circle trigonometry may assist in students' development of complex algebra as they transition to upper-division courses. Rectangular and polar coordinates are common in introductory courses and provide a context in which the distinctions between triangle and circle trigonometry can be discussed. The development of complex algebra skills, including changing between forms and selecting forms, relies on fluency in circle trigonometry. Relating coordinate systems may lessen the abstraction of complex numbers when students encounter complex algebra in middle-division physics courses.

\section{E. Future work}

Future work may aim to investigate the role of physical applications and tangible examples in students' understanding of complex numbers and functions. Because physics students use complex numbers and functions extensively to model the physical world, their perceptions and interpretations of these concepts may differ from students who encounter them only in mathematics contexts. Classical and quantum mechanics treat complex functions differently. The use of complex functions for mathematics simplification in classical mechanics starkly contrasts the complex algebra embedded in the mathematical structure of quantum mechanics. Students' development of complex algebra fluency and conceptions of forms and representations may be tied to the physical context, which may influence their treatments of complex algebra.

\section{ACKNOWLEDGMENTS}

This work was funded by NSF Grant No. DUE 1323800. We thank Dr. N. G. Holmes for helpful feedback on the manuscript.

\section{APPENDIX: FULL CODING OF STUDENTS' RESPONSES}

The boxes in Fig. 3 represent segmented portions of problem solving, discussed extensively in Ref. [20]. Each box represents portions of problem solving where neither the student nor interviewer interjects with an explicit reference to the goals outlined by the prompt. Specific errors appear in red with a letter corresponding to the error, and correctly performed steps are shown in black. The algebraic representations of $z$ are found in either the rectangular $(R)$, polar $(P)$, or exponential $(E)$ forms. Each instance of a resulting algebraic representation of $z$ is given by the abbreviation of the form and the parameters with which the form is expressed. For instance, the rectangular form in terms of $\ell$ and $c$, such as $z=\sqrt{\ell^{2}-c^{2}}-i c$, is represented as $R(\ell, c)$. Correct algebraic representations for the complex number are in black; incorrect algebraic representations are in red. 


\begin{tabular}{|c|c|c|c|c|}
\hline Student A & & & & \\
\hline & (a): $21 \mathrm{~T} 1 \mathrm{~T} 2 \mathrm{T3}$ & 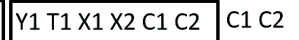 & P1 P2 & $\mathrm{T} 1 \mathrm{~T} 2$ \\
\hline & $\mathrm{Y} 1 \mathrm{X} 2 \mathrm{C} 2$ & & & \\
\hline
\end{tabular}

\begin{tabular}{|c|c|c|c|c|c|c|c|c|c|c|}
\hline \multirow{4}{*}{ Student B } & & \multirow{2}{*}{\multicolumn{2}{|c|}{ X2a Y1 Y2 Y1a Y2 }} & & & & & & \multirow[b]{2}{*}{ N2 } & \multirow[b]{2}{*}{ C3a } \\
\hline & (a): & & & $\mathrm{Z1}$ & $\mathrm{T} 4 / \mathrm{C} 1$ & $\mathrm{Z1}$ & $\mathrm{Z1C1}$ & Y2 N1 N1a & & \\
\hline & & & & & & & & & & \\
\hline & (b): & X1a & C3 Y2 N1 & Y1 C3 & N1 & Y1 C2 & $\mathrm{C} 2 \mathrm{a}$ & & & \\
\hline
\end{tabular}

Student C

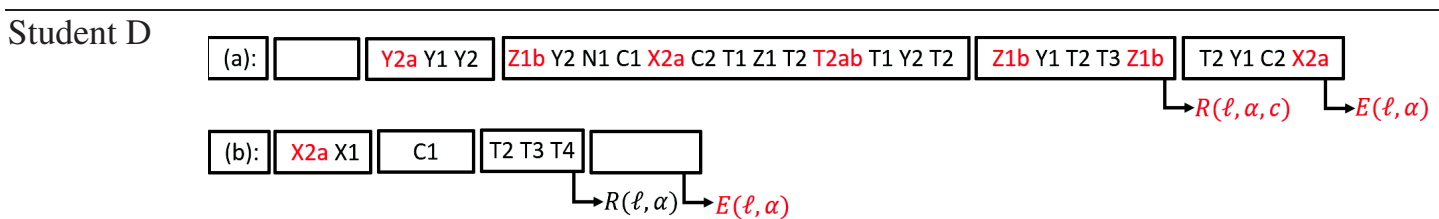

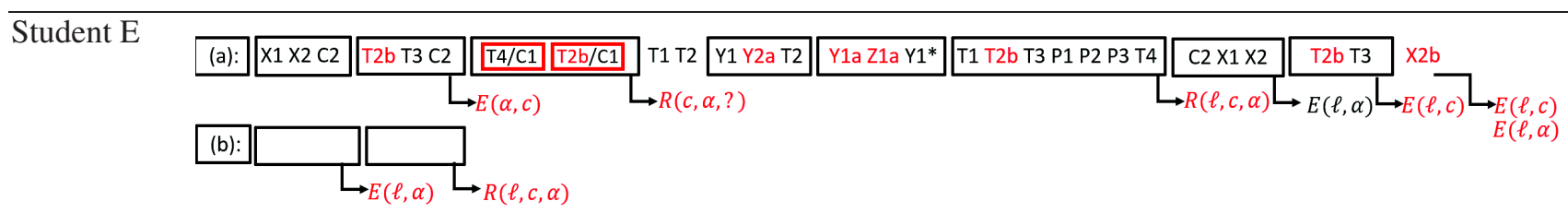

\begin{tabular}{|c|c|c|c|}
\hline Student F & & & \\
\hline & (a): & Y1 T2 X1 X2 C1 C2 & T1 T2 T2ab T2ab T1 T4 \\
\hline & (b): & Y1 X1 C1 X2 C2 & \\
\hline
\end{tabular}

\begin{tabular}{|c|c|c|c|c|c|c|}
\hline \multirow[t]{2}{*}{ Student $\mathrm{G}$} & & & \multirow{2}{*}{\multicolumn{2}{|c|}{$\mathrm{T} 1 \mathrm{~T} 2 \mathrm{~T} 1 \mathrm{Z} 1 \mathrm{~T} 1 \mathrm{~T} 2 \mathrm{~N} 1 \mathrm{a}$}} & \multirow[b]{2}{*}{ Y2 Y1 T2 T3 T1 T2 } & \\
\hline & (a): & Y2 N1 & & & & C1 ?? T1 T2 \\
\hline & (b): & & Z1 Y1 & T3 T4 & ?? & \\
\hline
\end{tabular}

FIG. 3. Codes are applied to students' interviews in groupings according to episodes where neither the interviewer nor student referred to the goals in the prompt. Tables II and III include descriptions of the codes. Correct steps are in black, and incorrect steps are in red. Resulting algebraic representations are given below the box by either rectangular $(R)$, polar $(P)$, or exponential $(E)$ form and by parameters provided by the prompt. Question marks indicate there is no corresponding code for significant parts of solving. Blank boxes indicate no relevant complex algebra manipulations were completed. Red boxes and slashes indicate that coded steps were blended in an incorrect combination. Full transcripts and corresponding coding can be found in Ref. [20]. *Interviewer provided information to the student. 
[1] V. A. Krutetskii, The Psychology of Mathematical Abilities in Schoolchildren (University of Chicago Press, Chicago, IL, 1976).

[2] H. Soto-Johnson, M. Oehrtman, and S. Rozner, Dynamic visualization of complex variables: The case of Ricardo in Proceedings of the 14th Annual Conference on Research in Undergraduate Mathematics Education (MAA, Portland, 2011), Vol. 2, p. 488.

[3] H. Soto-Johnson, M. Oehrtman, K. Noblet, L. Roberson, and S. Rozner, Experts' reification of complex variables concepts: The role of metaphor in Proceedings of the 15th Annual Conference on Research in Undergraduate Mathematics Education, Portland, 2012, edited by S. Brown et al. (MAA, Portland, 2012), Vol. 2, p. 189.

[4] M. E. Conner, C. Rasmussen, M. Zandieh, and M. Smith, Student understanding of complex numbers in Proceedings of the Conference on Research in Undergraduate Mathematics Education (Mathematical Association of America, San Diego, CA, 2007).

[5] P. Danenhower, Teaching and learning complex analysis at two British Columbia universities, Ph.D. thesis, Simon Fraser University, Burnaby, BC, CA, 2000.

[6] R. Nemirovsky, C. Rasmussen, G. Sweeney, and M. Wawro, When the classroom floor becomes the complex plane: Addition and multiplication as ways of bodily navigation, J. Learn. Sci. 21, 287 (2012).

[7] M. C. Nordlander and E. Nordlander, On the concept image of complex numbers, Int. J. Math. Educ. Sci. Technol. 43, 627 (2012).

[8] A. Panaoura, I. Elia, A. Gagatsis, and G. P. Giatilis, Geometric and algebraic approaches in the concept of complex numbers, Int. J. Math. Educ. Sci. Technol. 37, 681 (2006).

[9] G. Karakok, H. Soto-Johnson, and S. Dyben, Secondary teachers' conception of various forms of complex numbers, J. Math. Teach. Educ. 18, 327 (2015).

[10] H. Soto-Johnson and J. Troup, Geometric reasoning on the complex plane, in Proceedings of the 17th Annual Conference on Research in Undergraduate Mathematics Education, edited by T. Fukawa-Connolly, G. Karakok, K. Keene, and M. Zandieh (Mathematical Association of America, Denver, CO, 2014), p. 1044.

[11] H. Soto-Johnson and J. Troup, Reasoning on the complex plane via inscriptions and gesture, J. Math. Behav. 36, 109 (2014).

[12] A. Gupta, E. F. Redish, and D. Hammer, Coordination of mathematics and physical resources by physics graduate students, AIP Conf. Proc. 951, 104 (2007).

[13] M. E. Loverude and S. L. Li, Surprisingly, there is an actual physical application... Student understanding in math methods in Proceedings of the 2013 Physics Education Research Conference, Portland, OR, edited by P. V. Engelhardt, A. Churukian, and D. L. Jones (American Institute of Physics, Portland, OR, 2013), pp. 229-232.

[14] H. Sadaghiani, Conceptual and mathematical barriers to students learning quantum mechanics, Ph.D. thesis, Ohio State University, Columbus, OH, USA, 2005.

[15] H. Close, C. Schiber, E. Close, and D. Donnelly, Students' dynamic geometric reasoning about quantum spin-1/2 states in Proceedings of the 2013 Physics Education Research Conference, edited by P. V. Engelhardt, A. Churukian, and D. L. Jones (AIP, New York, 2013), pp. 93-96.

[16] T. Wan, P. Emigh, G. Passante, and P. Shaffer, Student understanding of period in introductory and quantum physics courses, in Proceedings of the 2016 Physics Education Research Conference, Sacramento, CA, edited by D. L. Jones, L. Ding, and A. Traxler (AIP, New York, 2016), pp. 380-383.

[17] E. M. Smith, J. P. Zwolak, and C. A. Manogue, Student difficulties with complex numbers in Proceedings of the 2015 Physics Education Research Conference, College Park, MD, edited by A. D. Churukian, D. L. Jones, and L. Ding (AIP, New York, 2015), pp. 311-341.

[18] P. Danenhower, Introductory Complex Analysis at two British Columbia Universities: The First Week-Complex Numbers, Research in Collegiate Mathematics Education VI, edited by F. Hitt, G. Harel, and A. Selden (American Mathematical Society, Providence, RI, 2006).

[19] K. Browne, A case study of how upper-division physics students use visualization while solving electrostatics problems, Ph.D. thesis, Oregon State University, Corvallis, OR, USA, 2001.

[20] E. M. Smith, Students' understanding of complex numbers in middle-division physics, Ph.D. thesis, Oregon State University, Corvallis, OR, USA, 2016.

[21] http://physics.oregonstate.edu/portfolioswiki/courses:start.

[22] B. G. Glaser and A. L. Strauss, The Discovery of Grounded Theory: Strategies for Qualitative Research (Aldine Publishing Co., Chicago, IL, 1967).

[23] B. D. Mikula and A.F. Heckler, Student difficulties with trigonometric vector components persist in multiple student populations in Proceedings of the 2013 Physics Education Research Conference, Portland, OR, edited by P. V. Engelhardt, A. D. Churukian, and D. L. Jones (AIP, New York, 2013), pp. 253-256.

[24] D. J. Ozimek, P. V. Engelhardt, A. G. Bennett, and N. S. Rebello, Retention and transfer from trigonometry to physics, AIP Conf. Proc. 790, 173 (2005). 